\title{
Two Ways Interaction between Lower Order Terms of Left Brain and Right Brain Cognitive Style and Relationship between Satisfaction and Turnover Intention
}

Abdul Halim Busari, PITAS/TASIC, Department of Organizational Development, FCSHD, Universiti Malaysia Sarawak, Kuhing, Malaysia

Yasir Hayat Mughal, Department of Organizational Development, FCSHD, Universiti Malaysia

Sarawak, Kuhing, Malaysia

\section{ABSTRACT}

This paper argues that teacher's job satisfaction should also have an impact on turnover intention. In this paper identified teacher's level of job satisfaction and turnover intention of advance learning institutions in the Khyber Pakhtoon Khwa Province of the Pakistan and the moderating effect of the cognitive style. Questionnaires were used to gather the information and 502 completed questionnaires were collected back from the respondents. The questionnaire was comprised of JDI consisting of seven items on job satisfaction, turnover Intention questionnaire consisting of three items and five point Likert scale was used and for cognitive style CSI, the cognitive style index was used, the fourth section was comprised of the open ended questionnaire and fifth section was comprised of demographic variables. Hierarchical multiple regressions have been used to check how much variance satisfaction shows upon turnover intention and also it has been checked that how much variance analysis and intuition cognitive style shows variance upon satisfaction and turnover intention of advance learning institutions of kpk province of Pakistan. The correlation results taken from bivariate Pearson correlation shows significant results which later on strengthen by the regression results. The regression results show that satisfaction shows variance upon turnover intention, this extended model of job satisfaction will be useful to bring the changes in the academician's satisfaction of Khyber Pakhtoon Khwa province of Pakistan. The findings of this study could be used to guide the advance learning institution's management and professional academicians build targeted learning activities around key components of the academician's job satisfaction, diagnose where people are in their journey, set personalize goals and provide feedback to management in the process of the development of policies for advance learning institutions academician's. In the context of the efficient and effective educational policy, a greater understanding of the academician's job satisfaction could facilitate the development of more effective policy, practice that would increase not only the job satisfaction of the academicians but decrease the turnover intention of the academician's.

\section{Keywords}

Analysis, Cognitive Style, HEIs, Intuition, Satisfaction, Turnover Intention

\section{INTRODUCTION}

A lot of issues has been there to look after by human resource management however; Organizational behavior is the most important factor which makes the management successful and also is the cause of management failure. Satisfaction is the most important issues for all academic researchers and also for professional researchers (Stacy, 1998; Locke \& Latham, 2000). The employees who are satisfied from 